\title{
INFLUENCE DE LA POLLUTION ORGANIQUE SUR LA FAUNE AQUATIQUE DES EAUX COURANTES (1)
}

\author{
par Paul VIVIER \\ (I.N.A.A. Station d'Hydrobiologie Continentale de Paris)
}

\begin{abstract}
De toutes les pollutions qui souillent nos cours d'eau, celles dues aux matières organiques rejetées par les agglomérations urbaines, les établissements d'élevage ou les usines agricoles sont de beaucoup les plus nombreuses. $\mathrm{Ce}$ sont d'ailleurs de plus en plus des pollutions mixtes complexes, où entrent en jeu, en dehors des produits habituels de dégradation de la matière organique, de nombreux composés chimiques dont l'utilisation se généralise: détergents, hydrocarbures, pesticides.
\end{abstract}

Les pollutions organiques au sens strict, objet de notre étude, se caractérisent par un appauvrissement marqué du milieu récepteur en oxygène, une augmentation de la turbidité, la présence fréquente de composés ammoniacaux ou phénoliques toxiques, de gaz carbonique, issus de la fermentation anaérobie, à cóté de matières nutritives non encore altérées (on trouve souvent des ablettes et des gardons au débouché des égouts). Elles donnent également des dépôts vaseux. La vase elle-même, non seulement a une très grande activité bactériologique, mais concentre des dizaines et des centaines de fois anions et cations qu'elle ne restitue à l'eau qui la surmonte que selon des modalités encore mal connues.

Au débouché d'un égout, le milieu récepteur est donc particulièrement complexe. Cependant à moins que le cours d'eau ne soit trop exigu pour des eaux résiduaires trop abondantes et qu'il y ait absence de courant, l'autoépuration au contact de l'oxygène dissous dans l'eau se fait naturellement plus ou moins bien, cela va sans dire, mais elle se fait, à moins qu'elle ne soit entravée par des produits surajoutés tels que les détergents, mais nous envi-

(1) Développement d'une communication préparée pour le colloque d'hydrobiologie organise par la Société zoologique de France à Dijon (mai 1968) qui, en raison des événements, n'a pu avoir lieu. 
sageons ici essentiellement des pollutions organiques classiques. La minéralisation des substances organiques est alors poussée jusqu'à l'oxydation et l'on aboutit finalement à la formation de nitrates, de sulfates et de phosphates.

Cette autoépuration par fermentation anaérobie puis aérobie est l'œuvre de bactéries, de champignons, d'algues, de protozoaires, de rotifères et de gastrotriches. L'oxygène provient des réserves apportées par le cours d'eau et aussi, progressivement, par les algues (Cladophora) et les macrophytes à chlorophylle dont le développement est accru par les sels nutritifs, nitrates et phosphates, à mesure de leur production. D'autres organismes en consommant des matières organiques favorisent cette autoépuration: ce sont notamment des Oligochètes, des Diptères, mais surtout la bactérie filamenteuse Sphaerotilus, facile à reconnaitre parce que ses colonies révèlent l'aspect de queue de mouton et forment dans le cours d'eau des plages souvent importantes, dès l'arrivée du rejet organique: on a estimé qu'un hectare de Sphaerotilus pourrait épurer une eau d'environ $3 \mathrm{~kg}$ de matières organiques par heure.

\section{ACTION DE LA POLLUTION ORGANIQUE SUR LA FAUNE}

L'absence ou la rareté de l'oxygène, la présence de gaz carbonique en quantité anormale, celle de composés toxiques, la turbidité de l'eau créent nécessairement des conditions de vie précaires pour les êtres vivants auxquels bien peu ne résistent. Pendant l'autoépuration, les conditions de l'environnement (oxygénation, etc.) varient; d'abord plus mauvaises au bout d'une certaine distance de la source de pollution qu'immédiatement après, elles vont ensuite généralement en s'améliorant de façon progressive; les êtres vivants ont dès lors plus ou moins de facilités à s'adapter à ces caractéristiques nouvelles. Du fait qu'il existe une certaine liaison entre le milieu pollué et les organismes aquatiques, on a songé à les utiliser comme indicateurs. Cette utilisation est-elle justifiée? C'est ce que nous nous proposons d'examiner. Nous distinguerons les protozoaires des macroinvertébrés.

\subsection{Les protozoaires.}

Les protozoaires jouent un rôle essentiel dans la destruction de la matière organique. Dans les zones très polluées, on observe tout d'abord quelques Flagellés tels que Bodo sp.; puis, dès que les conditions s'améliorent, des protozoaires mangeurs de bactéries: Colpidium colpoda, Glaucoma et Paramecium, Carchesium, Vorticella ; enfin des mangeurs d'algues: Chilodonella, Spirostomum, Stentor, Coleps, Didinium, Lionotus, etc..., accompagnés de rotifères; quand la minéralisation touche à sa fin, des Flagellès à chlorophylle en grand nombre, comme Euglena et Phacus.

Malheureusement, on ne peut guère, en pratique, utiliser les protozoaires comme indicateurs de pollution:

- des listes d'espèces ne suffisent pas; il faut qu'elles soient accompagnées du dénombrement des individus, certaines de ces espèces pouvant se trouver en quantité réduite dans des eaux non polluées;

- il est mal aisé d'avoir un échantilionnage valable des animaux microscopiques d'une rivière, eu égard à la grande diversité des microhabitats offerts par l'environnement ;

- les déterminations exactes sont le plus souvent difficiles mais nécessaires et exigent la présence de spécialistes; certains genres, tout au moins 
(Amoeba, Paramaecium, Trachelomonas, etc...), présentent, en effet, de grandes variations de sensibilité suivant les espèces. C'est pourquoi les protozoaires sont peu utilisés en pratique comme indicateurs.

\subsection{Les macroinvertébrés.}

II n'en est pas de même des métazoaires microscopiques généralement plus faciles à identifier. De nombreuses études ont été faites en Europe centrale, en Grande-Bretagne et aux Etats-Unis sur leur valeur indicatrice. Nous allons y puiser largement.

\subsubsection{Zone fortement polluée.}

Le premier effet de la pollution organique sur les macroinvertébrés par les nombreux facteurs limitants qu'elle amène, est de diminuer l'indice de diversité de la communauté considérée, c'est-à-dire le rapport du nombre d'espèces au nombre d'individus (ODUM 1960). De 27 en amont, les espèces tombent à 3 en aval dans le Kamazo (Michigan) (SURBER 1953); de 63 (1) à 9 dans le Lytle Creeck (GAUFIN et TARZWELL 1956) (fig. 1); pour les Coléoptères seuls, de 12 à 2 dans le White River (Indiana) (YOUNG 1961) (fig. 2).

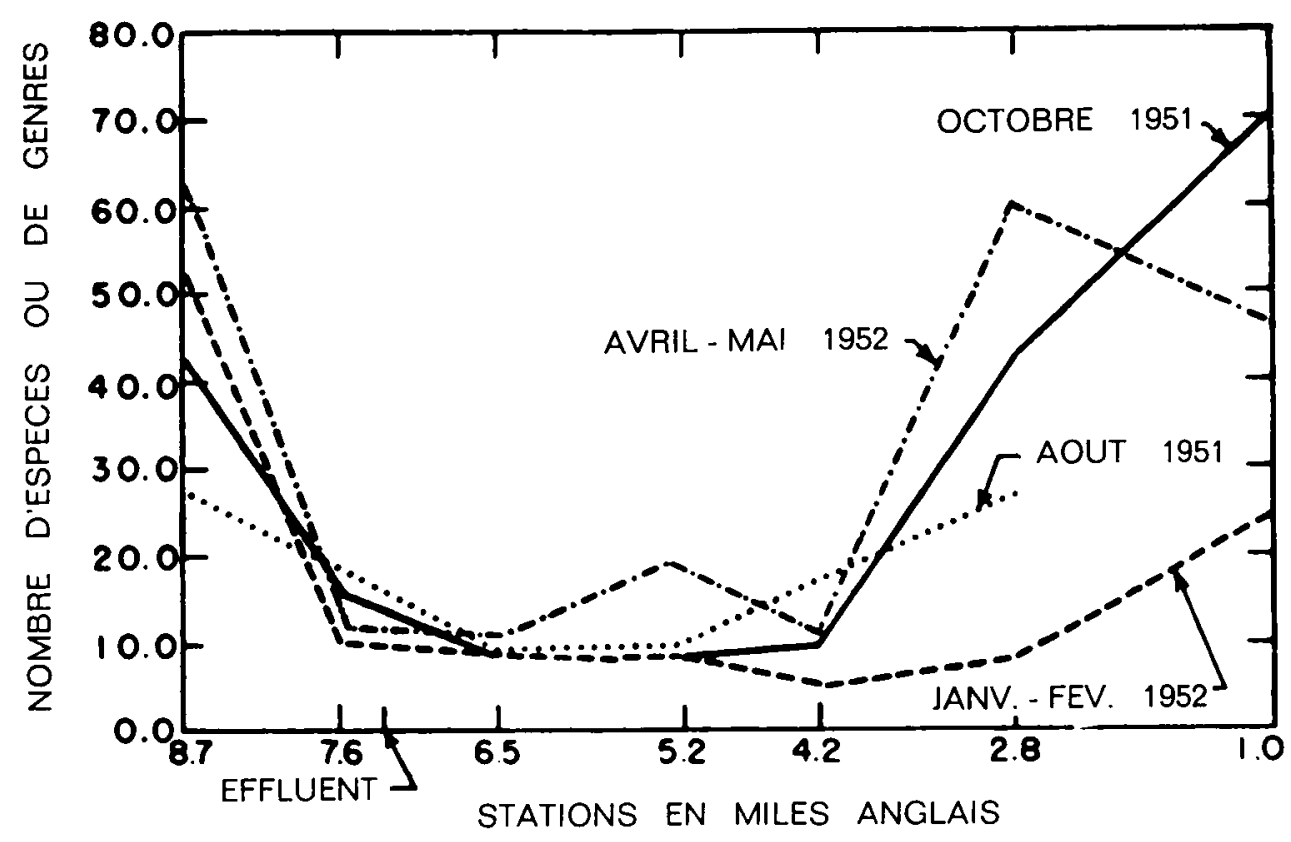

Fig. 1 - Distribution des macroinvertébrés, Lytle Creek.

L'effluent pollué affectant la rivière en amont comme en aval de son embouchure, seule la faune prélevée à deux miles en amont, a été jugée caractéristique d'eau claire (d'après GAUFIN et TARZWELL).

(1) Collemboles : 1 : Diptères : 18 ; Coléoptères : 13 ; Ephéméroptères : 4 ; Trichoptères : 6 ; Plécoptères : 3 ; Odonata : 4; Hémiptères : 3 ; Crustacés : 3 ; Mollusques : 4 ; Annelides : 1; Plathelminthes : 1 ; Nématodes : 2 . 
Par contre le nombre d'individus augmente énormément: 440000 Tubifex et Limnodrilus au $\mathrm{m}^{2}$ (RICHARDSON, 1929), 250000 Tubifex, 35000 larves de Culex, des larves d'Eristalis aussi nombreuses que "des herbes d'une prairie *. dans un autre cas (GAUFIN et TARZWELL).

Certains organismes seulement peuvent, en raison de leurs adaptations, supporter des teneurs très basses en oxygène (moins de $2 \mathrm{mg}$ par litre). $\mathrm{Ce}$ sont des Tubificides (Limnodrilus, Tubifex), des larves de Diptères pourvus d'un tube respiratoire (Psychoda, Eristalis, Culex); dès que l'oxygène augmente tant soit peu, des larves de Chironomus du groupe thumni de THIENEMANN 1954. I'hémoglobine qu'elles possèdent leur servant à transporter et à stocker l'oxygène (WALSHE, 1950). A Lytle Creeck, on trouvait également dans cette zone le Coléoptère Tropisternis natator, le Diptère Tabanus atratus, I'Hémiptère Hesperocorixa et le Pulmoné Physa integra.

\subsubsection{Zone intermédiaire ou de reconstitution progressive.}

A mesure que l'autoépuration se produit dans la rivière, la teneur en oxygène s'accroit, les conditions de vie s'améliorent et le nombre des espèces augmente. Mais ces conditions varient suivant les saisons, la température, les hautes et basses eaux, etc... si bien que les étendues respectives de la zone fortement polluée et de la zone de reconstitution qui lui succède sont variables ainsi que le nombre des espèces qui les peuplent. C'est ainsi que dans le Lytle Creeck, au débouché de l'égout, la D.B.O. 5 oscillait de 120 (déc. 1949, août 1950) à 20 ; l'oxygène dissous variait de 0 (déc. 1949 , août 1951) à $12 \mathrm{mg}$ (avril 1951). La rivière s'améliorait progressivement vers l'aval et, à $5 \mathrm{~km}$ au débouché de l'égout, la D.B.O. 5 s'était abaissée aux environs de 10 et les teneurs en oxygène étaient redevenues normales sans grandes variations.

C'est pourquoi si, dans la zone fortement polluée, le nombre des espèces passait, suivant les saisons, de 9 à 18, dans la zone de reconstitution (partie médiane), oscillait entre 9 (oct. 1956) et 32 (avril 1952); la communauté la plus caractéristique était faite là de quelques espèces de la zone fortement polluée confinées, en petit nombre de représentants, dans des microhabitats, et d'un nombre variable de formes les plus tolérantes des eaux propres, spécialement celles ayant une variété de moyens pour prélever de l'oxygène: des larves de Diptères (Simulium vittatum, Libellula sp., Tanypus stellatus), des nymphes d'Odonates, assez bien adaptées à une pauvreté relative en oxygène par leurs trachéo-branchies (on en a même rencontré dans des eaux où la teneur en oxygène tombait à $1 \mathrm{mg}$, toutefois pendant une courte période). Lorsque les conditions s'améliorent en avril, on trouvait alors 7 espèces appartenant aux trois groupes les plus sensibles à la pollution (Ephéméroptères. Plécoptères, Trichoptères) représentées par au moins 6 individus au $\mathrm{m}^{2}$, mais elles avaient disparu en automne.

Dans cette zone de reconstitution, on note, en outre, en Europe des larves de Mégaloptères (Sialis lutaria), de Chironomides de couleur variée (Tanytarsus, etc.), des sangsues, des mollusques (Sphaerium rivicola, Sphaerium corneum, Limnea pereger, Physa fontinalis), I'Isopode Asellus aquaticus (HYNES, 1960).

\subsubsection{Valeur indicative de différents groupes.}

\subsubsection{Ephéméroptères - Plécoptères - Trichoptères.}

Une biocénose formée de larves et nymphes d'Heptageniidés (Ephéméroptères), de Perlidés (Plécoptères) et de Trichoptères à fourreaux fixes, est 
indicatrice d'eau propre; son absence dénote d'ordinaire l'existence d'une pollution ou tout au moins d'un faible approvisionnement en oxygène.

Il faut se garder de conclusion trop hâtive car il existe chez les larves d'Ephéméroptères, de Plécoptères et de Trichoptères des variations importantes de résistance suivant les espèces, certaines pouvant même se trouver dans des zones où l'oxygène descend la nuit, à $1 \mathrm{mg}$ par litre. C'est le cas notamment des Ephéméroptères Stenonema femoratum et Callibaetis dans le Lytle Creek (GAUFIN et TARZWELL) (1), ainsi qu'en France de nombreux Baetidae (VERNEAUX) (2).

Dans ce même groupe, si les larves et nymphes d'Ameletus inopinatus, Rhitrogena hybrida, R. semicolorata, R. tatrica sont des indicateurs spécifiques d'eaux très pures ou pures, les larves des différentes espèces d'Ecdyonurus, d'Ephemerella, sont eurysaprobes. Baetis rhodani serait plus résistant à la pollution que $B$. pumillus.

Chez les Plécoptères, les larves et nymphes d'Arcynopteryx compacta, de Diura bicaudata seraient caractéristiques d'eaux très pures; il en serait de même pour Amphinemura borealis alors que Amphinemura sulcicollis se montrerait plus tolérant. Isoperla grammatica offrirait plus de résistance que Isoperla oxylepis et Isoperla rivulorum; Leuctra fusca estival le serait davantage que les autres espèces printanières de Leuctra; sans doute serait-elle mieux adaptée à résister à une période de basses eaux et de faible teneur en oxygène (ZELINKA et MARVAN, HYNES). Nemura cinerea offrirait plus de tolérance que $\mathbf{N}$. cambrica et $\mathbf{N}$. marginata (WINKLER, 1964), mais VERNEAUX (1969) (2) estime que les conclusions de ces différents auteurs sont discutables.

Chez les Trichoptères, si les larves et les nymphes d'Apatania fimbriata, Brachycentropus montanus, Drusus discolor, Agapetus sp. sont considérées généralement comme des indicateurs de pureté, celles de Glossosoma sp., Cheumatopsyche sp. supportent des milieux légèrement pollués; des larves d'Hydropsychidés ont été trouvées là où la teneur en oxygène tombait à $3 \mathrm{mg}$ par litre, mais elles restaient cependant localisées dans les portions les plus rapides du cours d'eau (ZELINKA et MARVAN, GAUFIN et TARZWELL); Hydropsche sp. a été lui-même observé dans des eaux nettement polluées, à D.B.O. supérieure à 20, et à très forte turbidité ( $1 \mathrm{~g}$ par litre) (ROBACK, 1962) (3).

\subsubsection{Coléoptères.}

Les Coléoptères adultes ont la possibilité d'accroitre la capacité d'air de leur système trachéen et de diffuser ce supplément par des mouvements de ventilation lorsqu'ils viennent respirer à la surface. A l'exception des Elmides (Stenelmis crenata, Stenelmis sexlineata p. ex.) qui vivent en eaux propres. ils peuvent être très résistants à la pollution. Il y a, là encore, de grandes variations suivant les espèces, si bien que l'on peut se servir d'une étude

(1) Les A. font remarquer qu'il est possible qu'il y ait eu un peu plus d'oxygène dans le lieu précis de récolte de ces deux espéces (radier ou faible profondeur) que dans le courant lui-même où il a été mesuré, ce qui souligne la difficulté de tirer des conclusions tant sont nombreux les microhabitats dans une surface restreinte de cours d'eau.

(2) Renseignement personnel. Je remercie $M$. VERNEAUX des observations qui lui ont été suggérées par la lecture de cette étude et dont j'ai tenu le plus grand compte.

(3) Sur 667 larves de Trichoptères observées appartenant à 20 genres, 117 ont été trouvées dans une turbidité de 10 ppm, 146 dans une de $25 ; 163$ pour $50 ; 156$ pour 100, 75 pour 500 et 10 pour 1000 . De même, sur 581 larves, 28 vivaient dans un D.B.O. de 0,1;83 dans un D.B.O. de 0,$5 ; 215$ dans un D.B.O. de $1 ; 205$ dans un D.B.O. de $5 ; 20$ dans un D.B.O. de $10 ; 28$ dans un D.B.O. de 20 et 4 dans un D.B.O. de 40 . 
de répartition de celles-ci pour diagnostiquer une altération d'un cours d'eau; leur nombre diminue d'une façon caractéristique en aval d'un effluent, comme le montrent les graphiques de diversité-abondance, donnant le nombre d'espèces différentes en fonction du pourcentage d'individus de chacune d'elle par échantillonnage (YOUNG, 1961) (1) (fig. 2).

\subsubsection{Diptères.}

Si les larves de Diamesa novorienda, Cricotopus absurdus, Calopsectra neoflavella, Liponeura sp. ne se rencontrent qu'en eaux riches en oxygène, la plupart des autres larves se trouvent partout, mais en plus grand nombre dans les eaux polluées riches en matières organiques, comme nous l'avons vu plus haut (2). Il y a là encore de grandes variations suivant les espèces, notamment chez les larves de Psychoda et de Pericoma, si bien qu'on a proposé de les utiliser comme indicateurs du degré de pollution d'une eau (VAILLANT, 1966).

\subsubsection{Crustacés inférieurs.}

Contrairement à ce que l'on admet généralement, les différentes espèces de gammares de nos régions, Rivulogammarus pulex, etc..., ne sont pas caractéristiques d'eaux pures. On les rencontre parfois dans des eaux moyennement polluées, mais elles ne sont jamais en grande quantité; la reproduction se fait mal. Asellus aquaticus, par contre, est un bon indicateur d'eaux polluées mais non très fortement (ZELINKA et MARVAN, HYNES).

\subsubsection{Mollusques.}

Certains mollusques, comme Bythinella austriaca, ne vivent que dans des eaux très riches en oxygène et par conséquent ont une grande valeur indicatrice. La plupart cependant d'entre eux se rencontrent dans des eaux moyennement polluées, comme Sphoerium rivicola et Sphoerium corneum. Le Pulmoné Physa integra qui facilement se ravitaille en oxygène peut vivre dans des eaux qui en sont presque dépourvues; il a été trouvé dans tout le cours du Lytle Creek, mais en plus grand nombre dans la zone polluée, plus nutritive. GAUFIN et TARZWELL le considèrent, lorsqu'il est associé à d'autres espèces, comme un indicateur valable de la pollution.

\subsection{Facteurs écologiques locaux.}

La réaction d'un organisme aux altérations d'environnement par pollution organique dépend, d'une part, de l'espèce, d'autre part du milieu. Or celui-ci n'est pas identique dans tous les cours d'eau ni pour un même cours d'eau, dans toutes les saisons; nous avons vu dans le Lytle Creek, combien pouvait varier, au débouché de l'égout pollueur, la D.B.O., la teneur en oxygène, le niveau des eaux, le débit. D'autres facteurs écologiques, variables avec les rivières ou les tronçons de rivière, en modifiant l'environnement, interfèrent avec les facteurs proprement dus à la pollution: altitude, $\mathrm{pH}$, dureté, nature du sol, érosion, pente, situation des affluents, présence de végétaux supérieurs, prédation, etc... Aussi les indications données par un organisme dans des conditions définies ne doivent pas être transposées sans discernement et sans étude préalable dans n'importe quel cours d'eau.

(1) Ne pouvant effectuer comme PRESTON, 1958, de grands échantillons représentés par une courbe logarithmique normale, YOUNG donne des graphiques dont l'allure rappelle la courbe classique nombre d'espèces - nombre d'individus d'ODUM. II suffit, d'après lui, de 50 à 100 spécimens.

(2) 38 espèces différentes de Tendipedidés ont été trouvées dans un D.B.O. maximum de 82 (CURRY, 1965), donc dans des eaux nettement polluées. 

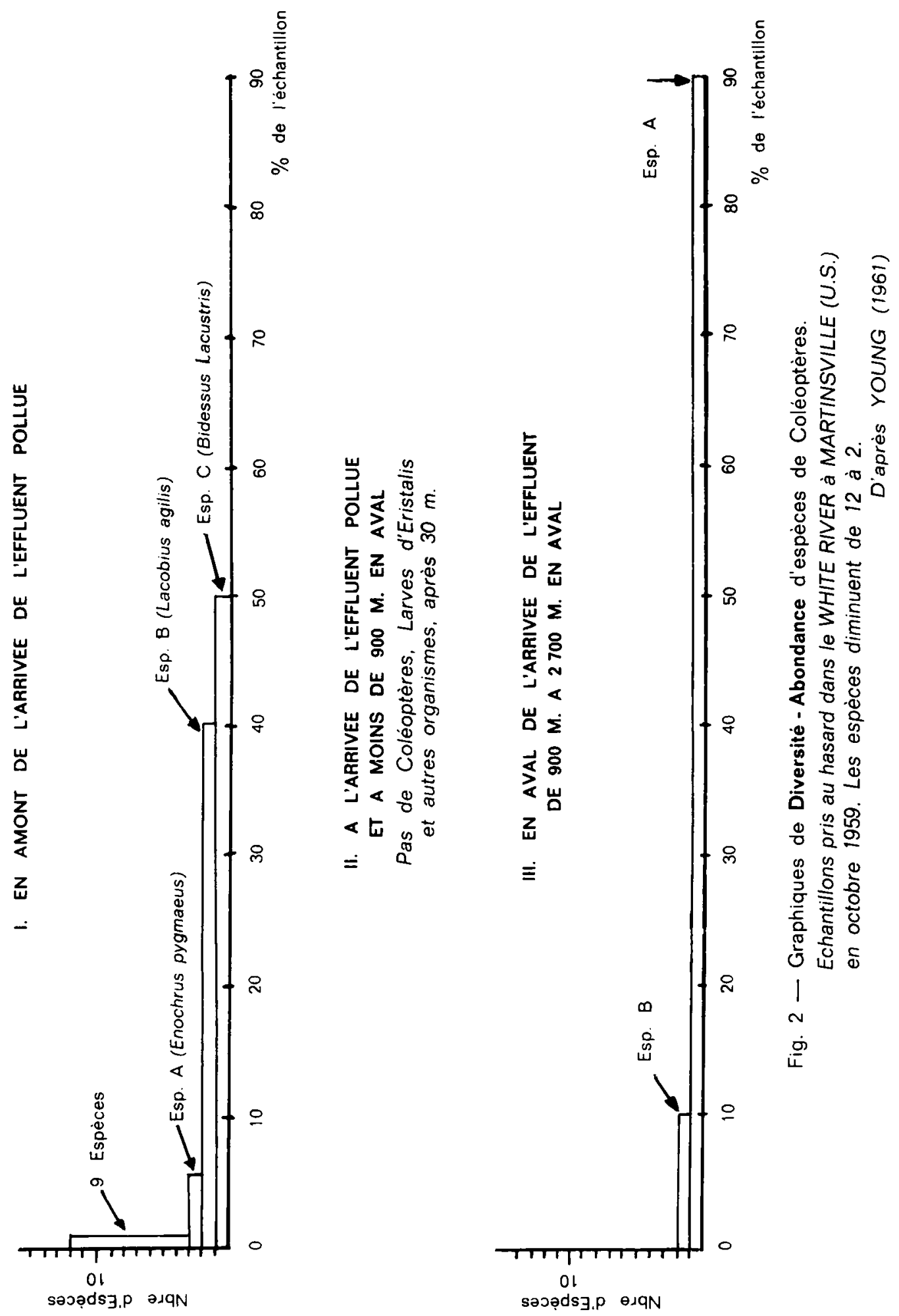


\section{LES ZONES DE SAPROBIES}

De ces différentes observations, il ressort que certaines espèces par le nombre de leurs représentants et encore mieux certaines associations sont théoriquement susceptibles dans des conditions normales de l'environnement, de caractériser la pureté ou la pollution de l'eau et pourraient donc servir d'indicateurs biologiques. C'est pourquoi, à la suite de KOLWITZ et MARSON, 1908, 1909, on a songé à répartir les espèces animales et végétales dans différentes zones correspondant à des étages différents de la pollution organique. En utilisant différents critères, physico-chimiques (quantité d'oxygène, degrés de minéralisation et d'oxydation des matières organiques, etc...), bactériologiques (nombre de germes au $\mathrm{ml}$ ) et biologiques (végétaux et animaux caractéristiques), on avait défini :

- une zone d'eau pure, riche en oxygène, appelée zone oligosaprobe. dans laquelle on a distingué parfois une zone d'eau très pure ou catarobe qu'on rencontre surtout dans les torrents de haute montagne près de leur source ;

- une zone moyennement polluée ou mésosaprobe, elle-même subdivisée en zones $\beta$ et $\alpha$, dans laquelle se range la quasi-totalité de nos cours d'eau de plaine, à a cyprinidés dominants * $\left(2^{\circ}\right.$ catégorie de la classification administrative) :

- une zone très polluée ou zone polysaprobe.

Le système des saprobies ainsi schématisé, longtemps utilisé en Europe centrale, a rendu des services. C'est ainsi que WINKLER, 1963, a pu répartir entre les différentes zones de saprobies, 54 espèces de larves et nymphes de Plécoptères de Tchécoslovaquie, d'après leur tolérance de plus en plus grande à la pollution. De son côté BERTHELEMY, 1966, après avoir comparé la répartition de 32 de ces espèces rencontrées d'amont en aval dans les torrents pyrénéens avec celle donnée par WINKLER, estime que * la similitude entre les deux classements est nette, les zones amont étant, en effet, les moins polluées .

Cependant le système de KOLKWITZ et MARSON n'est plus guère utilisé car il est l'objet de nombreuses critiques (GAUFIN et TARZWELL, 1956, HAVRANEK, 1966, ZELINKA et MARVAN, 1966, etc...). II ne saurait, en effet, résister sans de profondes retouches à un examen approfondi, tant d'ordre chimique que bactériologique et biologique. On a tenu compte surtout de la teneur en oxygène dans la classification, mais de nombreux autres facteurs, comme on a pu s'en rendre compte précédemment, causent une succession des biocénoses: relations entre espèces, substances organiques, nourriture offerte, nature du substrat, diminution de l'action toxique des produits de décomposition, etc... Comme l'écrivaient dernièrement ZELINKA et MARVAN, 1966. "le système des saprobies de KOLKWITZ et MARSON a un caractère très empirique... Du fait qu'il nous manque une définition satisfaisante des conditions physico-chimiques d'existence de la communauté à l'intérieur d'une zone saprobe et que cette définition ne peut encore être formulée, la liaison logique entre le caractère saprobe et la qualitè de l'eau fait défaut. Le système qui ne considère notamment ni la quantité totale de matières organiques dans le biotope considéré. ni le degré de dégradation de ces substances est donc très discutable. ” 


\section{MÉTHODES DE DIAGNOSTIC}

Pour apprécier, dans la pratique par examen de la faune et de la flore. la qualité biologique d'une rivière, dans ses rapports avec une pollution organique, plusieurs méthodes récentes ont été proposées. Nous en examinerons deux :

- celle de ZELINKA et MARVAN,

- celle de VERNEAUX et TUFFERY.

\subsection{Méthode de ZELINKA et MARVAN.}

Pour savoir où ranger une biocénose connue avec précision dans une portion de rivière donnée entre différents degrés de pureté, ZELINKA et MARVAN, 1960, dèterminent le nombre d'individus de chacune des espèces rencontrèes, les organismes les plus usuels des eaux douces étant dotés arbitrairement, dans un catalogue de 322 espèces animales et végétales publié par ces auteurs, de deux indices:

- l'un représentant la valeur indicative particulière i de l'espèce pour chacune des grandes zones suivantes: zone catarobe, zone oligosaprobe, zones mésosaprobes $\beta$ et $\alpha$, zone polysaprobe ;

- l'autre représentant une valeur indicative générale $g$. Les valeurs de $i$ sont chiffrèes de 1 à 10 , celles de $g$, de 1 à 5 . Les indications données par $\mathrm{i}$ et $\mathrm{g}$ se complètent, car plus une espèce est ubiquiste, moins sa valeur indicative générale est grande : un organisme eurisaprobe aura ainsi une valeur indicative générale faible, et réciproquement.

Prenons par exemple deux mollusques, l'un, ubiquiste. Ancylus fluviatilis, l'autre ne vivant que dans des eaux très pures et riches en oxygène, Bythinella austriaca. Les valeurs de $\mathbf{i}$ et de $\mathbf{g}$ données par le catalogue sont les suivantes:

\begin{tabular}{|l|c|c|c|c|c|c|}
\hline & $\begin{array}{c}\text { Zone } \\
\text { catarobe }\end{array}$ & $\begin{array}{c}\text { Zone } \\
\text { oligosaprobe }\end{array}$ & $\begin{array}{c}\text { Zones } \\
\text { mésosaprobes } \\
\beta\end{array}$ & $\begin{array}{c}\text { Zone } \\
\text { polysaprobe }\end{array}$ & $g$ \\
\hline $\begin{array}{l}\text { Ancylus fluviatilis } \ldots . . . \\
\text { Bythinella austriaca }\end{array}$ & 1 & 4 & 3 & 2 & & 1 \\
\hline
\end{tabular}

Pour chacune des espèces de la biocénose considérée, le nombre des individus récoltés $h$ est multiplié par la valeur indicative i', i', etc... de l'espèce dans chaque zone et par sa valeur indicative générale, $\mathbf{g}$ afin d'obtenir le produit gh $\left(i{ }^{\prime}+i^{\prime \prime}+i^{\prime \prime \prime}+i^{\prime \prime \prime}+i^{\prime \prime \prime \prime}\right)$, étant entendu que la somme $i^{\prime}+i^{\prime \prime}+i^{\prime \prime \prime}+$ $i " \prime+i " i^{\prime \prime \prime}$ est égale à 10 . On détermine ensuite pour chacune des zones, la valeur indicative moyenne $m$ de l'ensemble des espèces rencontrées; si celles-ci sont au nombre de $\mathbf{n}$ pour lesquelles les facteurs $h$, $i$ et $g$ ont chacun des valeurs déterminées $h_{1}, i_{1}, g_{1}, h_{2}, i_{2}, g_{2}, i_{n} h_{n} g_{n}$, cette valeur moyenne $m$ est égale au quotient des produits partiels $g_{1} h_{1} i_{1}, g_{2} h_{2} i_{2}$, etc... par la somme $S$ des produits $h_{1} g_{1}, h_{2} g_{2}$, etc... obtenus en multipliant le nombre d'individus $h_{1}, h_{2}$, etc... de chaque espèce par sa valeur indicative générale $g_{1}, g_{2}$, etc... On a ainsi pour chaque zone

$m=\frac{g_{1} h_{1} i_{1}+g_{2} h_{2} i_{2}+g_{3} h_{3} i_{3}+\ldots \text { etc. }}{S}=\frac{g_{1} h_{1} i_{1}+g_{2} h_{2} i_{2}+g_{3} h_{3} i_{3}+\ldots \text { etc. }}{g_{1} h_{1}+g_{2} h_{2}+g_{3} h_{3}}$ 
II est facile de vérifier que la somme des 5 moyennes indicatives correspondant aux 5 zones, $m_{1}+m_{2}+m_{3}+m_{4}+m_{5}$ est égale à 10 .

Prenons un exemple: dans une portion de rivière déterminée, on a récolté 24 espèces de mollusques, de Turbellariés, d'Amphipodes et de larves d'insectes dont on a dénombré les représentants. On a trouvé les moyennes indicatives générales suivantes:

\begin{tabular}{|c|c|c|c|c|}
\hline Z. catarobe & Z. oligosaprobe & Z. mé & robes & Z. polysaprobe \\
\hline & & $\beta$ & $a$ & \\
\hline 6,63 & 2,79 & 0,44 & 0,14 & 0 \\
\hline
\end{tabular}

Cette rivière doit donc être considérée comme non polluée organiquement. Cette méthode est intéressante, mais elle est longue; de plus l'attribution faite a priori de deux indices par espèce a un caractère arbitraire car elle suppose que soient parfaitement connues les exigences de chacune des dites espèces, ce qui n'est pas encore le cas.

\subsection{Méthode de VERNEAUX et TUFFERY.}

VERNEAUX et TUFFERY, 1967, ont proposé une méthode pratique inspirée par celle utilisée en Grande-Bretagne par le Trent River Autority (1964). Elle est simple, non spécifique d'un type donné de pollution ni d'une catégorie de cours d'eau (par là même applicable à l'ensemble du réseau hydrographique) ; elle présente une sensibilité et une précision suffisante pour être standardisée et permettre l'exploitation codifiée des résultats.

Pour les raisons indiquées au paragraphe $1-1$, les auteurs n'ont retenu comme matériel que les macroinvertébrés benthiques et, au lieu de baser les résultats sur l'identification des espèces, ce qui nécessiterait le concours d'un grand nombre de spécialistes, ils étudient les variations de la composition globale des biocénoses benthiques, ce qui leur permet d'évaluer l'aptitude biologique du cours d'eau considéré basée sur la diversité, l'abondance et la nature globale de son peuplement.

Cette appréciation de la qualité biologique globale d'une eau se fait conventionnellement par la determination d'un indice biotique dont la valeur s'échelonne entre 0 et 10.

Dans les trois échantillons recueillis par prélèvement, on détermine d'abord le groupe zoologique dominant, c'est-à-dire le groupe faunistique de référence de la biocénose. Puis l'on dénombre le nombre total d'unités systématiques présentes dans chaque groupe. L'unité systématique devrait être en principe l'espèce, seule unité écologique valable. Mais comme, d'après ce qui a été dit plus haut, la détermination rigoureuse de l'espèce est pratiquement impossible, l'unité retenue sera donc selon les ordres *l'espèce, le genre, la tribu, la sousfamille ou la famille; d'une manière générale, elle sera arrêtée à la limite de la détermination simple, le plus souvent au genre, l'essentiel n'étant pas la taxonomie spécifique exacte, mais de dénombrer le plus grand nombre d'unités systématiques différentes dans chaque groupe *.

Dans le tableau ci-contre (fig. 3), la composition des groupes faunistiques formant échelle est établie en fonction de la sensibilité décroissante des dominants à la pollution générale du milieu, les plus sensibles étant placés le plus 
TABLEAU STANDARD DE DETERMINATION DES INDICES BIOTIQUES

\begin{tabular}{|c|c|c|c|c|c|c|c|c|}
\hline \multicolumn{4}{|c|}{$\begin{array}{c}1 \\
\text { Groupes Faunistiques }\end{array}$} & \multicolumn{5}{|c|}{$\begin{array}{c}\text { III } \\
\text { Nombre total des unités systématiques } \\
\text { présentes }\end{array}$} \\
\hline \multirow{4}{*}{1} & \multirow{4}{*}{$\begin{array}{l}\text { Plécoptères ou } \\
\text { Ecdyonuridae }\end{array}$} & \multirow{4}{*}{$\begin{array}{l}1 \\
2\end{array}$} & \multirow{4}{*}{$\begin{array}{l}\text { + d'une U.S." } \\
\text { I seule U.S. }\end{array}$} & $\begin{array}{c}\text { de } \\
0 \text { à } 1\end{array}$ & $\begin{array}{c}\text { de } \\
2 \text { à } 5\end{array}$ & $\begin{array}{l}\text { de } \\
6 \text { à } 10\end{array}$ & $\begin{array}{c}\text { de } \\
11 \text { a } 15\end{array}$ & $\begin{array}{c}\text { de } \\
16 \text { et } t\end{array}$ \\
\hline & & & & \multicolumn{5}{|c|}{ Indice biotique } \\
\hline & & & & - & 7 & 8 & 9 & 10 \\
\hline & & & & 5 & 6 & 7 & 8 & 9 \\
\hline \multirow[t]{2}{*}{2} & Trichoptères & 1 & + d'une U.S. & - & 6 & 7 & 8 & 9 \\
\hline & à Fourreaux & 2 & 1 seule U.S. & 5 & 5 & 6 & 7 & 8 \\
\hline \multirow{2}{*}{\multicolumn{2}{|c|}{$\begin{array}{l}\text { Ancylidae } \\
3 \text { Ephéméroptères } \\
\text { sauf Ecdyonuridae }\end{array}$}} & 1 & \multirow{2}{*}{$\begin{array}{l}+ \text { de } 2 \text { U.S. } \\
2 \text { ou - de } 2 \text { U.S. }\end{array}$} & 一 & 5 & 6 & 7 & 8 \\
\hline & & 2 & & 3 & 4 & 5 & 6 & 7 \\
\hline 4 & $\begin{array}{l}\text { Aphelocheirus } \\
\text { Odonates ou } \\
\text { Gammaridae ou } \\
\text { Mollusques (sauf } \\
\text { Sphaeridae) }\end{array}$ & 0 & $\begin{array}{l}\text { Toutes les U.S. } \\
\text { ci-dessus absentes }\end{array}$ & 3 & 4 & 5 & 6 & 7 \\
\hline 5 & $\begin{array}{l}\text { Asellus ou } \\
\text { Hirudinae ou } \\
\text { Sphaeridae ou } \\
\text { Hémiptères (sauf } \\
\text { Aphelocheirus) }\end{array}$ & 0 & $\begin{array}{l}\text { Toutes les U.S. } \\
\text { ci-dessus absentes }\end{array}$ & 2 & 3 & 4 & 5 & - \\
\hline 6 & $\begin{array}{l}\text { Tubificidae ou } \\
\text { Chironominae des } \\
\text { Groupes Thumni- } \\
\text { plumosus }\end{array}$ & 0 & $\begin{array}{l}\text { Toutes les U.S. } \\
\text { ci-dessus absentes }\end{array}$ & 1 & 2 & 3 & - & - \\
\hline & Eristalinae & 0 & $\begin{array}{l}\text { Toutes les U.S. } \\
\text { ci-dessus absentes }\end{array}$ & 0 & 1 & 1 & - & $一$ \\
\hline
\end{tabular}

* U.S. Unité systématique choisie.

Fig. 3 - Détermination des indices biotiques par la méthode de VERNEAUX et TUFFERY. 
haut. Puis est déterminé le nombre total d'unités systématiques présentes, sans qu'il soit tenu compte du nombre d'individus, celui-ci pouvant varier en dehors de toute pollution, sous l'action de nombreux facteurs, notamment de la saison ou de la nature des supports.

Cette opération est effectuée dans chacun des deux facies du cours d'eau, le facies lotique (eaux courantes) et le facies lentique (eaux calmes).

A partir des groupes faunistiques de référence et du nombre d'unités systématiques rencontrées, le tableau ci-contre permet de déterminer rapidement l'indice biotique.

Prenons des exemples. Dans un prélèvement, on a dénombré dix-sept unités systématiques dont quatre appartiennent au groupe faunistique des Trichoptères à fourreaux, les Plécoptères étant absents. On se reporte au 2-1 de la colonne I et la colonne III donne immédiatement la valeur de l'indice biotique : 9.

Dans un autre prélèvement, il est compté neuf unités systématiques, aucune d'entre elles n'appartenant aux quatre premiers groupes faunistiques de référence de la colonne $\mathrm{I}$. On est naturellement conduit au $n^{\circ} 5$ de la colonne 1 (Asellus, Hirudinées, etc...), groupe effectivement présent; la colonne III indique que l'indice biotique est de 4 .

Le groupe faunistique supérieur de référence, le nombre total d'unités systématiques et l'indice biotique sont codés pour chacun des 2 faciès lotique et lentique et les variations d'indice d'un prèlèvement là un autre sont représentées graphiquement sur un parcours de plusieurs kilomètres (fig. 4). "Si les points $A$ et $B$ ne sont pas trop éloignés l'un de l'autre (moins de $10 \mathrm{~km}$ ), s'ils sont dans la même zone écologique, si la nature géologique, la granulométrie, la couverture végétale des deux faciès sont à peu près identiques, si la température des deux points est voisine, s'il n'y a pas confluence entre $A$ et $B$ et que $\Delta A, B \geqslant-|2|$ (différence d'indice entre $A$ et $B$ ), on peut conclure à l'existence d'une pollution lorsqu'au moins l'un des indices est $<5$. * Le point amont étant toujours pris comme référence, il $y$ a pollution si $\triangle A B$ est négatif; au contraire, si $\triangle A B$ est positif, le milieu devient plus favorable. Plus $\Delta A B$ est grand en valeur absolue, plus la pollution est importante.

On remarquera, dans le graphique ci-contre, que, à partir du point dont la valeur est la plus basse, plus les angles $\alpha_{c}$ et $\alpha_{1}$ du graphique des indices biotiques sont grands, plus vite la rivière retrouve son aspect normal, à la condition, bien entendu, que son débit soit constant tout au long de la zone polluée.

Afin de limiter autant que possible une interprétation subjective de la valeur indicielle et de ne pas notamment attribuer à des interventions extérieures, des phénomènes dus à des conditions naturelles défectueuses, les valeurs des indices en chaque point sont confrontées avec les composants de l'environnement.

Cette méthode qui ne s'applique pas uniquement aux pollutions organiques classiques a été adoptée par le Ministère de l'Agriculture en 1967 pour l'inventaire des pollutions de cours d'eau; elle s'est révélée d'un bon usage dans la pratique, mais son amélioration n'est pas exclue. 


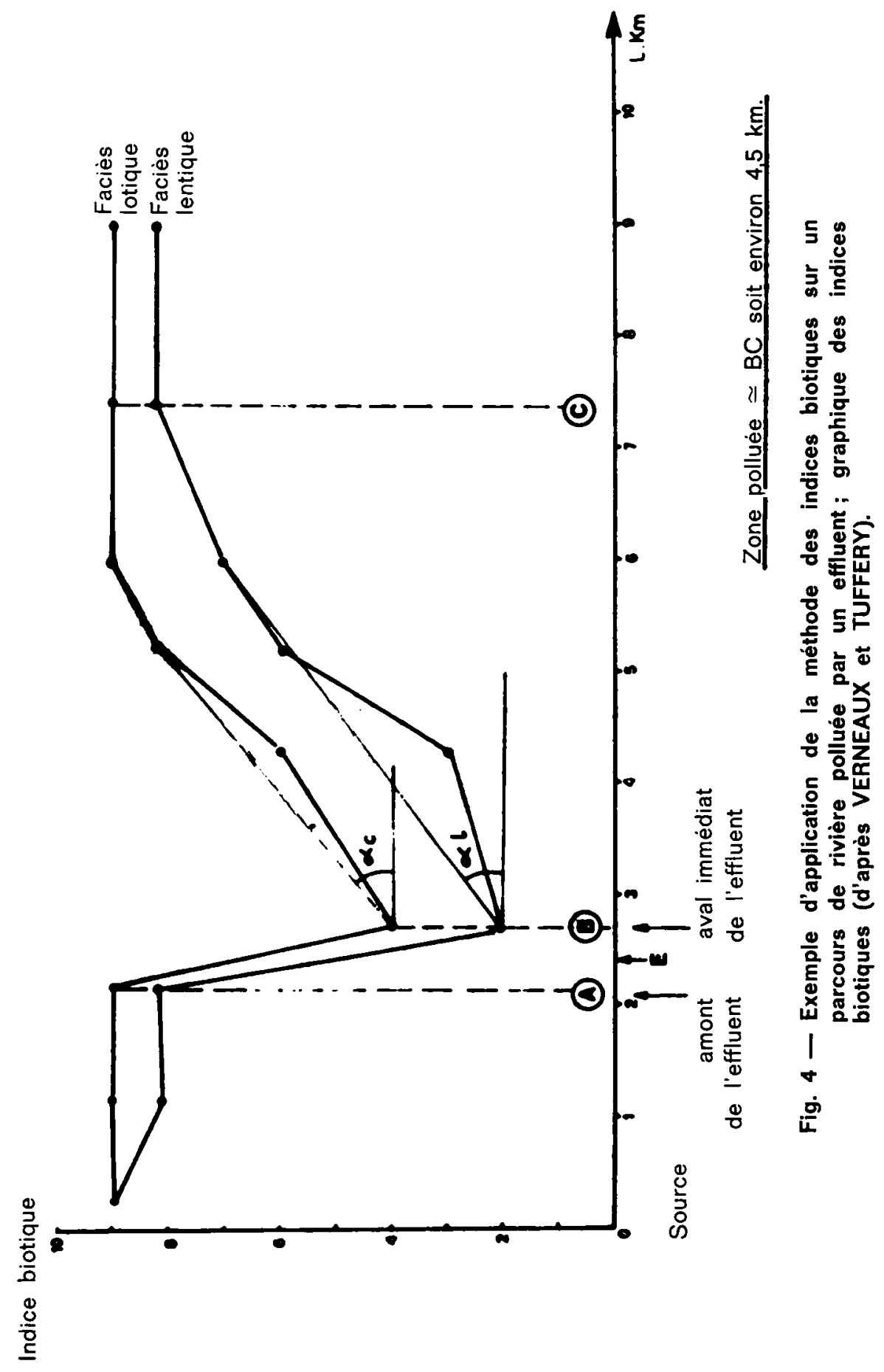




\section{CONCLUSIONS}

1) Les associations, plus que les espèces dites "caractéristiques " prises isolément, donnent des indications valables sur le degré de pollution. La présence d'une de ces espèces, sans indication du nombre d'individus à l'unité de surface, n'est nullement significative. Les espèces de la zone polluée peuvent se rencontrer dans des microhabitats de la zone propre, mais elles se trouvent en petit nombre. De même des organismes sensibles à la pollution sont susceptibles de vivre en hiver et au printemps lorsque les eaux sont hautes, la température basse et le degré d'oxygénation élevé dans des eaux relativement polluées, mais leur présence n'est que momentanée: ils ne sont jamais en grande quantité et s'y reproduisent assez mal.

2) Un certain nombre de facteurs écologiques locaux peuvent modifier l'environnement et par conséquent la valeur indicative d'une espèce et même celle d'une association: érosion, variations de débit, nature du sol, situation des affluents, prédation, etc... On doit en tenir compte si l'on veut diagnostiquer convenablement une pollution par des indicateurs biologiques.

3) Le degré de sensibilité à la pollution variant souvent avec les espèces d'un même genre, il importe de procéder à des déterminations rigoureuses, ce qui n'est pas toujours aisé et demande généralement la présence de nombreux spécialistes.

4) Les conclusions de certains travaux anciens sont entachées d'erreur, dues à des méthodes défectueuses d'échantillonnages et de prélèvements. Ces études doivent être remises en chantier. C'est ainsi, pour ne citer qu'un exemple, que pour dénombrer les organismes vivant dans la vase, comme les Tubifex, les appareils de récolte doivent pénétrer à au moins $11 \mathrm{~cm}$ (BRINKHURST, 1966).

5) Les travaux à réaliser au laboratoire et sur le terrain pour préciser la sensibilité réelle à la pollution organique de nombre d'organismes, demandent des équipes de chercheurs spécialisés, ce qui rend leur réalisation difficile. C'est ainsi que l'étude de GAUFIN et TARZWELL, 1956, a été faite en collaboration avec les sections de bactériologie, de chimie-physique, de biologie du Robert Taft Sanitary Engineering Center, le grand Centre d'études de la pollution de Cincinnati et l'aide de 11 spécialistes pour la détermination des espèces.

6) II est cependant possible, au point de vue pratique, d'utiliser la faune aquatique dans le diagnostic des pollutions organiques en se servant de l'une ou l'autre des méthodes indiquées dont l'intérêt est réel et qui, à l'usage, devront être améliorées. 


\section{BIBLIOGRAPHIE SOMMAIRE}

BERTHELEMY C. (1966) : Recherches écologiques et biogéographiques sur les Plécoptères et Coléoptères d'eau courante (Hydraena et Elminthidae) des Pyrénées. Annls Limnol., 2, 2, 227-458.

BERTRAND H. (1954): Les insectes aquatiques d'Europe, I, 558 pp., II, 547 pp., Paris.

BRINKHURST R.D. (1966): The Tubicidae (Oligochaeta) of polluted waters. Verh. int. Verein. theor. angew. Limnol., 16, 854-859.

BUTCHER R.W., LONGWELL J. and PENTELOW F.T.K. (1937): Survey of the river Tees, III. The non-tidal reaches chemical and biological. Tech. Pap. Wat. Pollut. Res. Lond., 6, $187 \mathrm{pp}$.

CHOUTEAU F. (1968): Influence de la pollution industrielle et domestique sur les populations animales de la rivière Isère au cours de sa traversée de la région grenobloise. Trav. Lab. Hydrobiol. Piscic. Univ. Grenoble, 59-60, 39-63.

CURRY L.L. (1965): A survey of environmental requirements for the midge (Diptera: Tendipedidae). Biological problems in water pollution. Third seminar, Cincinnati, 127-141.

GAUFIN A.R. and TARZWELL C.M. (1956) : Aquatic macro-invertebrate communities as indicators of organic pollution in Lytle Creeck. Sew. industr. Wastes, 28, 906-924.

HAVRANEK M. (1966) : Einige Bemerkungen zur Theorie des Kokwitz-Marsonchen Saprobiensystems vom chemischen Standpunkt gesehen. Verh. int. Verein. theor. angew. Limnol., 16, 823-829.

HYNES H.B.N. (1960): The biology of polluted waters. Liverpool, $202 \mathrm{pp}$.

KOLKWITZ R. und MARSON R. (1908): Okologie der pflanzlichen Saprobien. Ber. Dt. Bot. Ges., 26 a, 195-229.

KOLKWITZ R. und MARSON R. (1909): Okologie der tierischen Saprobien. Int. Revue Ges. Hydrobiol. Hydrogr., 2, 126-152.

LIEBMANN H. (1960-1962): Handbuch der Frischwasser und Abwasserbiologie I, 2* édit., 588 pp., II, 1149 pp., Munich.

MACAN T.T. (1963) : Freshwater ecology. London, 388 pp.

ODUM E.P. (1959) : Fundamentals of ecology. Philadelphia and London, $2^{\circ}$ édit., $546 \mathrm{pp}$.

PRESTON F.W. (1958): Analysis of the Audubon Christmas counts in terms of the logonormal curve. Ecology, 38, 4.

ROBACK S.S. (1965) : Environmental requirements of Trichoptera. Biological problems in water pollution. Third Seminar, Cincinnati, 118-126.

SURBER E.W. (1953): Biological effects of pollution in Michigan waters. Sew. industr. wastes, $25,79-86$. 
THIENEMANN A. (1954): Chironomus. Binnengewässer, 20, 834 pp.

TUFFERY (G.) et VERNEAUX (J.) (1967): Méthode de détermination de la qualité biologique des eaux courantes. Exploitation codifiée des inventaires de la faune de fond. Trav. Sect. Tech. P. et P., C.E.R.A.F.E.R., Paris, 23 pp.

VAILLANT F. (1967): Utilisation des Diptères Psychodidae comme indicateur de certains caractères des eaux courantes. Verh. int. Verein. theor. angew. Limnol., 16, $1721-1725$.

VERNEAUX J. et TUFFERY G. (1967): Une méthode zoologique pratique de détermination de la qualité biologique des eaux courantes. Indices biotiques. Annls scient. Univ. Besançon, Zoologie, 3, 79-90.

VERNEAUX J. (1969): Recherches sur une méthode pratique d'étude synthétique des cours d'eau. Application à la rivière Doubs - Thèse, Faculté des Sciences de Besançon, 153 pp. Ronéotypé.

VIVIER P. (1967) : Influence de la pollution des eaux sur la flore et la faune piscicoles. Evolution et modification du milieu. Bull. tech. Infor., Ministère de l'Agriculture, 224, pp. 221-230.

WINKLER D. (1964) : Ueber die praktische Bedeutung der Plecopteren. Gewäss. Abwass. 34/35 (Verh Z. int. Symp. Plecopteren), 131-138.

WIEBE A.H. (1927): Biological survey of the upper Mississipi river with special reference to pollution. Bull. U.S. Bur. Fish., 43, 137-167.

ZELINKA M. et MARVAN P. (1961): Zur Präzisierung der biologischen Klassifikation der Reinheit fliessender Gewässer. Arch. Hydrobiol., 57, 3, 389-407.

ZELINKA M. et MARVAN P. (1966) : Bemerkungen zu neuen Methoden der saprobiologischen Wasserbeurteilung. Verh. Internat. Verein theor. angew. Limnol., 16, 817-822. 